\title{
La place de la grammaire dans les manuels de la méthode directe : quelques exemples (1896-1930)
}

The place of grammar in direct method textbooks: some examples (1896-1930

Irene Valdés Melguizo

\section{OpenEdition}

Édition électronique

URL : https://journals.openedition.org/dhfles/7511

DOI : $10.4000 /$ dhfles. 7511

ISSN : 2221-4038

Éditeur

Société Internationale pour l'Histoire du Français Langue Étrangère ou Seconde

Édition imprimée

Date de publication : 1 décembre 2020

Pagination : 135-160

ISSN : 0992-7654

Référence électronique

Irene Valdés Melguizo, « La place de la grammaire dans les manuels de la méthode directe : quelques exemples (1896-1930) ", Documents pour l'histoire du français langue étrangère ou seconde [En ligne], 64-65 | 2020, mis en ligne le 05 mars 2021, consulté le 25 mars 2023. URL : http://

journals.openedition.org/dhfles/7511; DOI : https://doi.org/10.4000/dhfles.7511

Ce document a été généré automatiquement le 25 mars 2023.

Tous droits réservés 


\title{
La place de la grammaire dans les manuels de la méthode directe : quelques exemples (1896-1930)
}

The place of grammar in direct method textbooks: some examples (1896-1930

\author{
Irene Valdés Melguizo
}

\section{Introduction}

1 Dans cette étude, nous analysons une série de manuels qui accompagnent la méthode directe (désormais MD) en s'interrogeant sur la place que la grammaire y occupe. Car, comme nous le verrons, cette MD «ne néglige pas la grammaire, et [...], tout en renonçant au thème, elle arrive à enseigner la correction grammaticale par des procédés à la fois pratiques et méthodiques » (Schweitzer $1903: 31$ ).

2 Comme nous l'avons constaté dans une autre étude (Suso López \& Valdés Melguizo 2020), la progression de la grammaire se fait principalement à travers le vocabulaire, donc la grammaire et le vocabulaire vont de pair dans cette méthode (Sweet 1900, Schweitzer 1903, Rochelle 1906, Bénédict 1950, Schweitzer \& Simonnot 1917). L'analyse de notre corpus confirme cette tendance. La MD s'éloigne, ainsi a priori de la présentation canonique de la grammaire de la méthode traditionnelle (désormais MT), où la grammaire occupait la place la plus importante, voire la seule place dans l'enseignement/apprentissage de la langue (désormais e/a) (Germain 1993 : 101-102).

3 Pour notre analyse nous avons sélectionné une série d'ouvrages publiés entre 1896 et 1930 représentants de la MD. Notre liste n'est pas exhaustive mais, à notre avis, il s'agit d'un échantillon assez représentatif de ce courant méthodologique :

a)- S. Alge (51896a). Leçons de français, première année ;

5 (51896b). Leçons de français, deuxième année ;

6 b)- S. Alge \& W. Rippmann ( $\left.{ }^{9} 1904\right)$. Leçons de français basées sur les tableaux de Hölzel ; 

étrangère (désormais LE), de la part de nombreux chercheurs (Fernández Fraile \& Suso López 1999, 2008 ; García Bascuñana 2016, Suso López 2016) qui ont étudié diverses questions concernant ces ouvrages: les contextes sociaux et historiques, les aspects biographiques... Notre objectif est de compléter ce qui a déjà été écrit à leur sujet en y étudiant plus spécifiquement la place de la grammaire. d'approche grammaticale présent dans ces manuels de la MD; et (ii) analyser la progression grammaticale à l'intérieur de ces ouvrages du français LE pour un public scolaire (avec quelques exemples d'exercices de ces manuels).

\section{La méthode directe : lignes générales}

Les précurseurs de la MD seraient, selon Reinfried (1990 : 126-156) Basedow et Wolke (le mouvement philantropiste de Dessau de la fin du XVIII ${ }^{\mathrm{e}}$ siècle) et Wilhelm Viëtor (1882) considéré comme l'initiateur de la Réforme, avec son ouvrage Der Sprachunterricht muss umkehren. Ein Beitrag zur Ueberbürdungsfrage, où ce méthodologue rompt aussi avec la MT (Bénédict 1950 : 13-14).

La phrase conclusive de l'ouvrage de la MD de Bénédict (1950) représente explicitement une des principales lignes générales de cette méthode : Prima docens utile sapientia (la première sagesse est d'enseigner ce qui est utile). Cette maxime se retrouve dans tous les manuels de notre corpus. L'e/a d'une LE doit être préalablement utile pour l'acquisition d'un mode de communication efficace (Germain 1993 : 127).

Une autre caractéristique de la méthode concerne « le terme 'direct' référant à l'accès 'direct' au sens étranger, sans l'intermédiaire de la traduction, de manière à amener l'apprenant à penser directement dans L2 » (Germain 1993 : 130). Il s'agit du rejet de la langue maternelle (désormais LM) des apprenants au profit du développement de la LE même au début de l'e/a de cette nouvelle langue, autrement dit, de prendre de la distance vis-à-vis d'une approche bilingue pour favoriser l'approche unilingue (Besse $2012: 12)$.

19 Selon Schweitzer, le rôle joué par la LM quand on apprend une LE serait la cause de difficultés dans le processus d'acquisition de cette nouvelle langue. La traduction, par conséquent, viendrait compliquer le processus d'e/a de la LE :

Si [...], je recours à la traduction, si je dis : le mot Apfel signifie en français pomme,

l'opération se compliquera d'une équation nouvelle ; au lieu d'une équation à deux 
termes, nous aurons l'équation à trois termes suivante : Apfel $=$ pomme $=0$; au lieu d'une opération intellectuelle, nous en aurons deux. [...] C'est donc la traduction qui est la voie la plus longue; elle est représentée par une ligne brisée, alors que le procédé par intuition est figuré par une ligne droite (Schweitzer 1903 : 7).

D'ailleurs, comme nous le vérifierons dans notre corpus, dans la MD la traduction est complètement interdite et les apprenants sont obligés de penser (ou au moins ils doivent essayer de penser), directement dans cette LE. Mais, parfois comme l'affirment Schweitzer \& Simonnot, les explications en LM peuvent être utiles pour l'e/a de la grammaire et du vocabulaire :

il faut reconnaître, en effet, que la condition essentielle de tout enseignement grammatical est la clarté, et ce serait faire preuve d'une intransigeance intempestive que de bannir absolument l'emploi de la langue maternelle pour ce genre d'explications, alors que nous l'avons admise dans certains cas pour l'interprétation du vocabulaire (Schweitzer \& Simonnot 1917 : 163-164). et même dans les explications grammaticales :

n'oublions pas enfin que les explications grammaticales reviennent fréquemment au cours d'une classe; négliger de les donner en langue étrangère, quand on le peut, c'est se priver volontairement d'un excellent moyen d'exercer les élèves dans cette langue (Schweitzer \& Simonnot $1917: 165-166)$.

Ces affirmations nous font donc poser la question suivante : pourquoi faut-il négliger la LM des apprenants lorsque l'on veut leur faire comprendre des questions, a priori difficiles pour eux? L'idéal serait d'utiliser principalement la LE comme langue d'explication et de discours et si les apprenants trouvent des difficultés avoir aussi recours à leur propre LM. Nous serions ici alors dans une interprétation plus ouverte du caractère 'direct' de cette méthode.

MD met l'accent précisément sur l'oralité de la langue répondant au besoin d'apprendre une langue pour pouvoir s'exprimer: «le but général de la méthode directe est d'apprendre à utiliser la langue pour communiquer: c'est pourquoi l'apprenant doit apprendre non seulement à répondre aux questions mais à en poser " (Germain 1993 : 128).

Il y a donc rupture méthodologique avec la MT ; la MD naît comme une réaction au système pédagogique de la MT. La MD s'inscrit ainsi «dans la foulée des mouvements ou initiatives de réforme contre la grammaire-traduction, considérée comme inefficace, calquée sur les langues mortes, et orientée vers l'écrit » (Germain 1993 : 127). Et comme l'affirme Reinfried: «Les méthodologistes directs avaient déploré la monotonie de l'enseignement par la méthode synthétique de grammaire et de traduction » (Reinfried 1999 : 7).

la MD, comme l'affirme Suso (2016) est « un organismo vivo (que crece, que se desarrolla), y tangible físicamente (mediante su forma oral : cf. las leyes fonéticas) que una comunidad establece como medio de comunicación " (2016: en ligne). Nous pouvons donc y observer une différence avec la conception de la langue préconisée par la MT, où celle-ci était considérée comme «medio de expresión del pensamiento » (ibid. : en ligne).

Cette MD est ainsi, plus proche de la méthode dite naturelle: «this revolt against method was further led to an advocacy of the 'natural method' by children learn their own language » (Sweet 1899: 75). Même si la MD prétend renouer avec ce modèle plus naturel de l'e/a, elle n'est pas sans inconvénient, selon Sweet : 
so imperfect is this natural method, that even with the help of school-training and the incessant practice of every life, very few ever attain a really thorough mastery of their own language. When we say that any one is 'eloquent', or that he 'has a good style', or 'is a good speaker', or 'can tell a story very well', we hardly mean more than that of his fellows (Sweet $1899: 75$ ). dans le matériel utilisé dans cette MD. Nous constatons, dans notre corpus, que les images, les affiches, bref, tout l'aspect visuel, prend une place dominante. Le Tableau mural pédagogique devient « l'auxiliaire indispensable du professeur de langues vivantes [...] dont nous ne pourrons bientôt plus nous passer, quand nous aborderons les groupes d'idées n'ayant pas de rapport avec la classe » (Rochelle $1906: 10$ ).

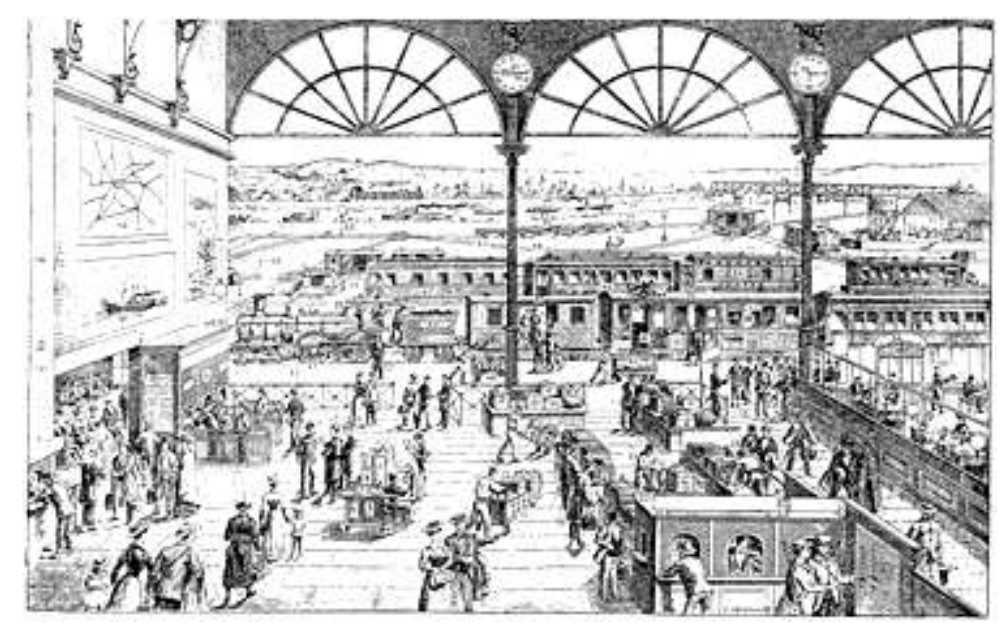

Document 1 : Exemple de Tableau mural pédagogique (Rochelle 1906 : 36)

Ce tableau mural permet d'associer des images aux concepts sans recourir à la traduction: «l'avantage principal [...] est d'associer la mémoire des yeux à celle de l'oreille, sans faire intervenir ni la traduction ni la même forme écrite du mot " (Rochelle 1906 : 19). Cette association aide les apprenants à découvrir le vocabulaire de la nouvelle LE. Les types d'affiches proposées sont les suivantes : «affiches de tourisme, de réclames, de plans de villes, de cartes géographiques, de journaux illustrés, [qui] contribueront à fixer les connaissances en mémoire » (Bénédict 1950 : 17).

30 Ce matériel, comme l'indique Jespersen, doit s'adapter au niveau des apprenants: «quieren que haya progreso gradual en la dificultad, es decir, que el material de enseñanza debe estar graduado desde las cosas más fáciles a las que vayan siendo cada vez más difíciles » (Jespersen 1925 : 326).

\section{La grammaire et le vocabulaire}

31 Nous verrons de quelle façon le vocabulaire et la grammaire sont les piliers de cette MD, et ces deux concepts y seront en symbiose, tel que l'assuraient Schweitzer \& 
Simonnot $(1917: 169)$ : «La méthode directe, loin de négliger la grammaire, réussit à enseigner la correction grammaticale par des procédés aussi vivants et aussi rationnels que ceux qui ont présidé à l'étude du vocabulaire ».

Le vocabulaire joue alors, un rôle très important dans l'e/a de la LE dans la MD. Le fondement psychologique sur lequel se fonde l'assimilation de cet élément sera l'associationnisme :

The psychological foundation of the practical study of languages is the great law of association, to which we have frequently had occasion to allude already. The whole process of learning a language is one of forming associations. When we learn our own language, we associate words and sentences with thoughts, ideas, actions, events (Sweet 1899 : 103).

Et selon cet auteur pour y arriver, les étapes nécessaires à l'acquisition du vocabulaire à travers l'associationnisme sont :

The following are the main axioms of the principles of association: (1) Present the most frequent and necessary elements first; (2) Present like and like together; (3) Contrast like with unlike till all sense of effort in the transition ceases; (4) Let the associations be as definite as possible; (5) Let the associations be direct and concrete not indirect and abstract; (6) Avoid conflicting associations (Sweet 1899 : 105-109).

Toutes ces lignes générales peuvent se résumer ainsi : aller du plus simple au plus complexe, du facile au difficile, c'est-à-dire, ce qui sera, en fin de compte, le plus proche de l'apprenant pour arriver à la réalité la plus éloignée de celui-ci : « il paraît logique de commencer notre enseignement par l'intuition de la réalité qui nous entoure. Nous nommerons les objets et les personnes de la salle, en les désignant au moyen du geste indicatif, le plus élémentaire des mouvements d'expression » (Schweitzer 1903 : 9).

Jespersen représente aussi ces mêmes principes à l'heure d'organiser le vocabulaire :

Podría ser provechoso hacer una colección sistematizada de material léxico; no, claro es, un diccionario común, ya que el orden alfabético es casi de lo más antimétodico que puede haber, sino un vocabulario preparado con inteligencia [...]. Debería, de todos modos, ser más pequeño y contener sólo palabras que fuesen realmente necesarias (Jespersen 1926 : 9).

Et ensuite, dans une deuxième étape, il faudrait se servir des images (ou des Tableaux muraux pédagogiques): "pour amener dans la classe les choses du dehors, nous recourrons aux images et aux collections. Il serait désirable que chaque établissement possédât une ou plusieurs salles réservées à l'enseignement des langues et munies du matériel nécessaire » (Schweitzer $1903: 9$ ).

En ce qui concerne la place de la grammaire, elle présentera un changement radical par rapport aux méthodologies antérieures.

Dans la méthode directe, en effet, la grammaire, que l'on prétendait bannie de notre enseignement, joue un rôle prépondérant. Elle est notre régisseur général. Mais, comme tout bon régisseur, elle se tient dans la coulisse. Elle fait appliquer le règlement en se montrant le moins possible (Rochelle 1906 : 30).

Le nouvel ordre de présentation se construit autour du vocabulaire: «le vocabulaire occupe une place plus importante que la grammaire» (Germain 1993:129); et du plus simple au plus complexe : «non plus dans l'ordre arbitraire des dix parties du discours, cher aux grammairiens, mais bien dans l'ordre naturel, qui va du plus simple au plus complexe » (Rochelle 1906: 8), et également comme l'affirme Schweitzer \& Simonnot 
(1917: 122) : «les promoteurs sérieux de la réforme ont affirmé la nécessité d'un enseignement grammatical mené de front avec celui du vocabulaire ».

L'approche privilégiée pour l'e/a de la grammaire sera l'approche inductive, c'est-àdire, d'abord les exemples, ensuite la règle : «tout le monde est d'accord aujourd'hui pour appliquer à l'enseignement grammatical la méthode inductive qui consiste à extraire les lois du langage des faits observés. C'est ce qu'exprime le précepte bien connu: Il faut enseigner la grammaire par la langue et non la langue par la grammaire » (Schweitzer \& Simonnot $1917: 124-125)$.

La règle grammaticale sera apprise, ainsi, par les apprenants grâce à cette démarche :

nous leur avons fait découvrir cette règle par la comparaison d'une série d'exemples appropriés, et nous n'avons formulé la règle en dernière analyse que comme la constatation d'un fait souvent noté. Suivant une jolie formule : La règle n'est plus pour nous que l'expression consciente d'une habitude consciente (Rochelle $1906: 35$ ).

41 Jespersen, à son tour, utilise le terme de "Gramática inventiva » quand il fait référence à cette approche inductive : « en la cual se lleva constantemente al alumno a descubrir por sí mismo los teoremas y las demostraciones » (Jespersen 1925 : 368).

Par rapport à la progression de la grammaire, le processus à suivre sera l'augmentation de la difficulté grammaticale, à travers les exercices de grammaire :

Il passe avec le plus grand soin des phrases simples du début à des formes de langage de plus en plus complexes. Et c'est précisément dans cette gradation minutieuse des formes du langage que réside le principe fondamental de l'enseignement grammatical. Il ne servirait à rien d'entasser chapitres de mots sur chapitres de mots : la grammaire est le fil conducteur qui doit traverser tous nos exercices de langage du commencement à la fin. Ces exercices seront donc rigoureusement ordonnés et gradués (Schweitzer \& Simonnot 1917 : 122-123).

Parfois, certains méthodologues de l'époque, tels que Viëtor (1882) ou Jespersen (1904), ont reproché l'absence de la grammaire dans la MD. De fait, et bien au contraire, la grammaire y est présente : «la méthode directe n'est donc pas, comme on le prétend parfois, incapable d'enseigner la grammaire. Nous dirons même mieux : elle est la seule façon à la fois logique et facile d'enseigner la grammaire » (Schweitzer $1903: 17$ ). Mais elle n'occupe plus la place centrale donc: "elle s'intégrera aux autres ressources linguistiques qui l'étayeront et qu'elle éclairera » (Bénédict 1950 : 27).

Bénédict fait même mention du développement de l'instinct grammatical grâce à la MD : «La méthode directe tend à éveiller, à provoquer chez l'étudiant des tendances qui, peu à peu, engendrent l'instinct grammatical, le développent par l'exercice empirique, en délestant l'élève de l'encombrant appareil traditionnel » (Bénédict 1950 : 27).

Nous constatons dans notre corpus tous ces principes de la MD que nous venons de rappeler.

\section{La place de la grammaire dans notre corpus}

La composition des ouvrages analysés montre exactement ce fil conducteur de la MD. Les caractéristiques centrales et habituelles sont les suivantes: la langue explicative utilisée dans les manuels sera la langue française (donc, refus direct de la LM) (Alge, Rippmann, Camerlynck \& Camerlynck, Valette, Alge \& Rippmann, Schweitzer \& 
Simonnot), l'utilisation des images (Alge, Rippmann, Camerlynck \& Camerlynck, Valette, Alge \& Rippmann, Schweitzer \& Simonnot), et le plus important pour notre étude, la progression grammaticale accompagnée par le vocabulaire (Alge, Rippmann, Camerlynck \& Camerlynck, Schweitzer \& Simonnot, Alge \& Rippmann, Valette).

Nous allons présenter ainsi ces caractéristiques propres aux manuels analysés en nous centrant d'abord sur les images et le vocabulaire, et ensuite, sur la grammaire et le vocabulaire.

\section{Les images et le vocabulaire}

Comme nous avons pu le constater, la MD se sert principalement de l'exploitation des images dans le processus d'e/a de la LE. En conséquence, les manuels examinés utilisent ce moyen pédagogique.

En ce qui concerne le manuel de la première année d'Alge, l'usage de l'image pour travailler le vocabulaire est préconisé par cet auteur parce que «le terme étranger se lie directement, immédiatement à l'idée éveillée par la vue de l'objet lui-même » (Alge ${ }^{5} 1896 \mathrm{a}: \mathrm{V}$ ). Toutes les petites leçons de ce manuel tournent autour des gravures (doc. 2). Alge construit ainsi toutes les leçons à partir de ces images. Il s'agit à chaque fois d'une description minutieuse de chaque gravure. Nous constatons donc, de quelle façon le vocabulaire, et conséquemment la grammaire, avance au fur et à mesure de la description faite de l'image, du plus simple (la famille, le verbe être, les animaux...) au plus complexe (les professions, la nourriture...). Chaque image proposée par Alge représente une saison : le printemps, l'été, l'automne, l'hiver.

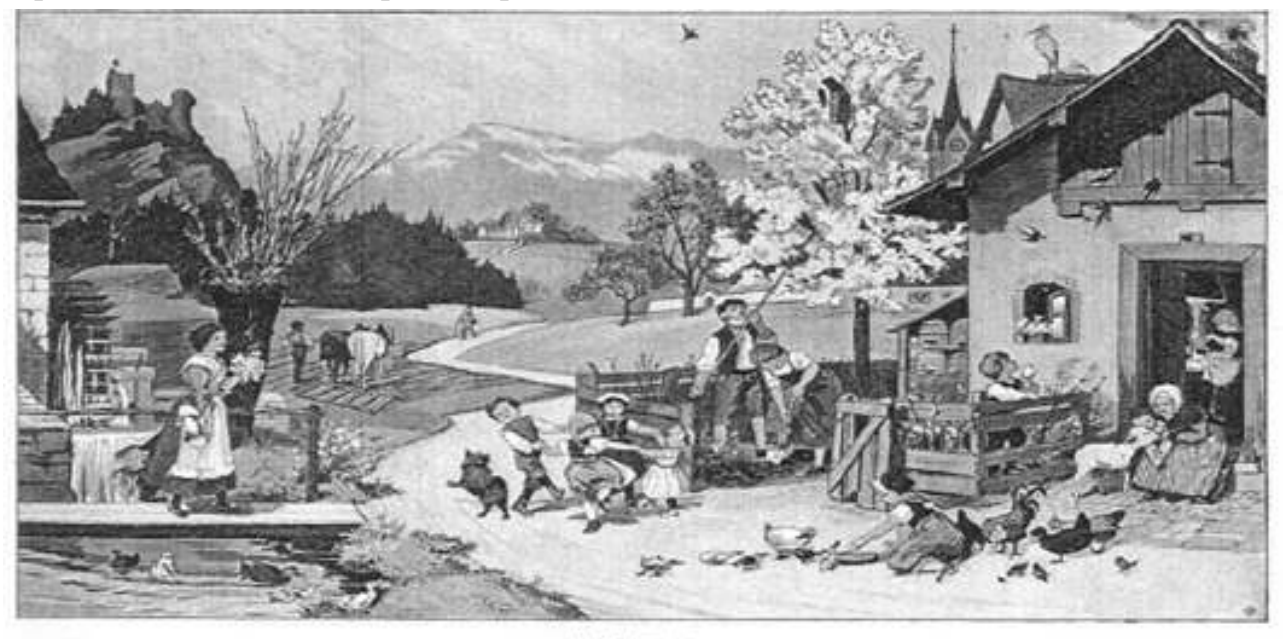

Pristemps.

Document 2 : Alge (51896a : 59)

Les activités mises à disposition pour travailler ces images sont, d'abord, une série de phrases à répéter, et ensuite, une série de questions pour appliquer le vocabulaire présenté dans les phrases précédentes: 


\section{5.}

Le chien est ntile; il garde lia maison. Les abeilles sont dans les ruches; elles rolent de flour en Heur ou elles ramassent le miel. Le miel est doux. Les aheilles travaillent toujours; elles sont laborienses, elles ne sont pas oisives.

Questions. Qu'est-ce que les abeilles? la ruche? Qu'est-ce que le chieu garde? Qui est-ce que la grand mère garde? Qu est-ce que les abeilles ramassent? Comment est le miel? Comment sont les abeilles? Comment sappelle la dememre des aheilles? des poules? des étourneaux? Oi rulent les abeilles? Ou sont les ruches de M. Auguste Bontemps? Combien de ruches y a-t-il derrière la maison? Qni est-ce qui ramasse des papiers? Qui est-ce qui mange le miel? Aimez-rous le miel? Qui est-ce qui n'est pas oisif?

Document 3 : Alge ( $\left.{ }^{5} 1896 a: 59\right)$

Pour ce qui est du vocabulaire, Camerlynck \& Camerlynck conseillent d'utiliser ce moyen pédagogique: «[...] les objets eux-mêmes, les tableaux dessinés, les gestes, actions et mouvements, etc.; nous avons pour notre part multiplié les illustrations et les indications à l'usage des élèves pour le travail à la maison» (Camerlynck \& Camerlynck $1921:$ IV).

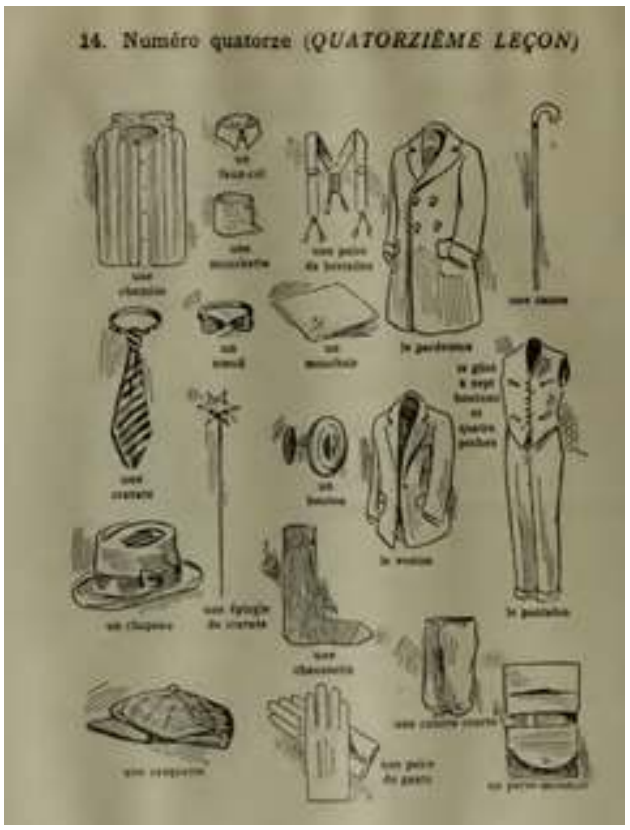

Document 4 : Camerlynck \& Camerlynck (1921 : 28) 
52 À titre d'exemple, les activités présentes pour acquérir ce vocabulaire sont les activités de conversation. L'objectif principal sera de réemployer le vocabulaire présent dans l'image qui ouvre la leçon :

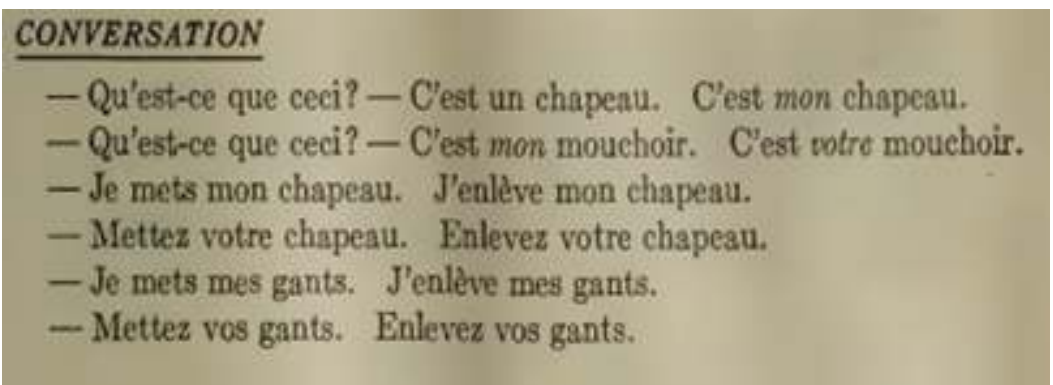

Document 5 : Camerlynck \& Camerlynck (1921 : 29)

53 D'un autre côté, les gravures sont accompagnées d'exercices de questions-réponses sur la description de la gravure, et également sur la grammaire présentée sur ces gravures :

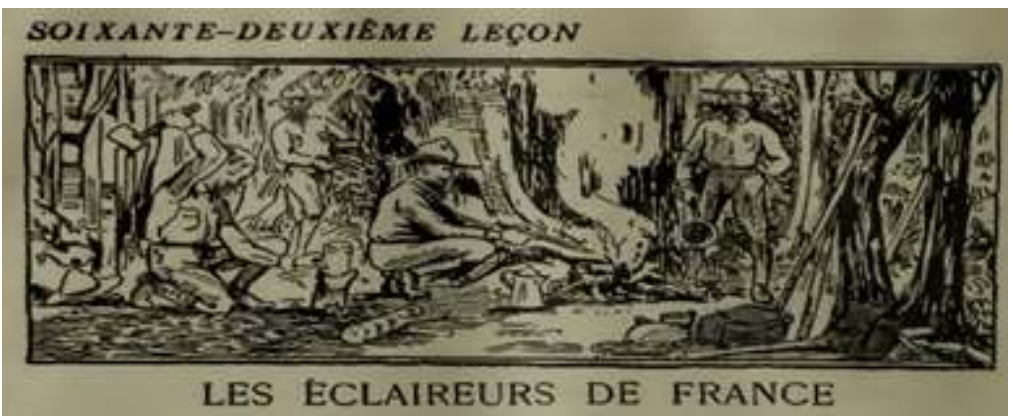

Document 6 : Camerlynck \& Camerlynck (1921: 118)

\begin{tabular}{|c|c|}
\hline \multicolumn{2}{|c|}{ DEVOIR } \\
\hline 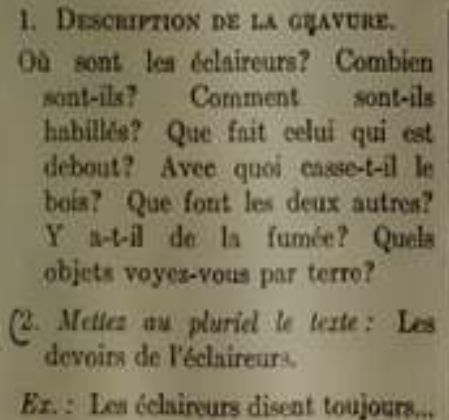 & 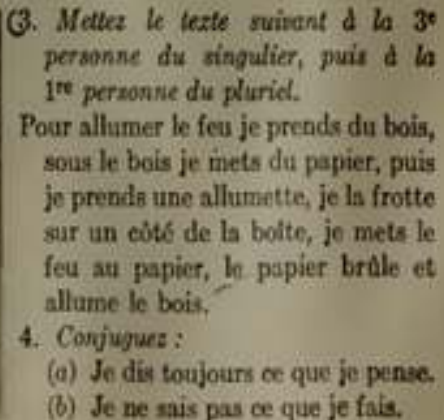 \\
\hline
\end{tabular}

Document 7 : Camerlynck \& Camerlynck (1921 : 119)

Quant aux manuels de Rippmann (First steps et Further steps), les images y sont bien évidemment utilisées. Les leçons sont organisées à partir de ces images. Les activités à faire avec ces images sont principalement des questions-réponses sous forme de dialogues où les notions grammaticales sont soulignées pour que les apprenants puissent s'y concentrer. 


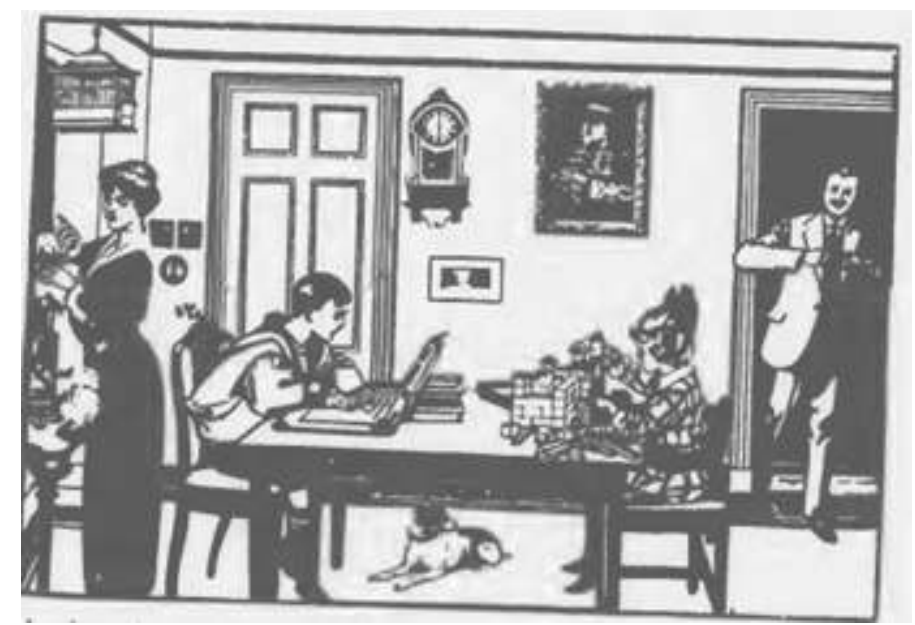

Document 8 : Rippmann $(1915: 22)$

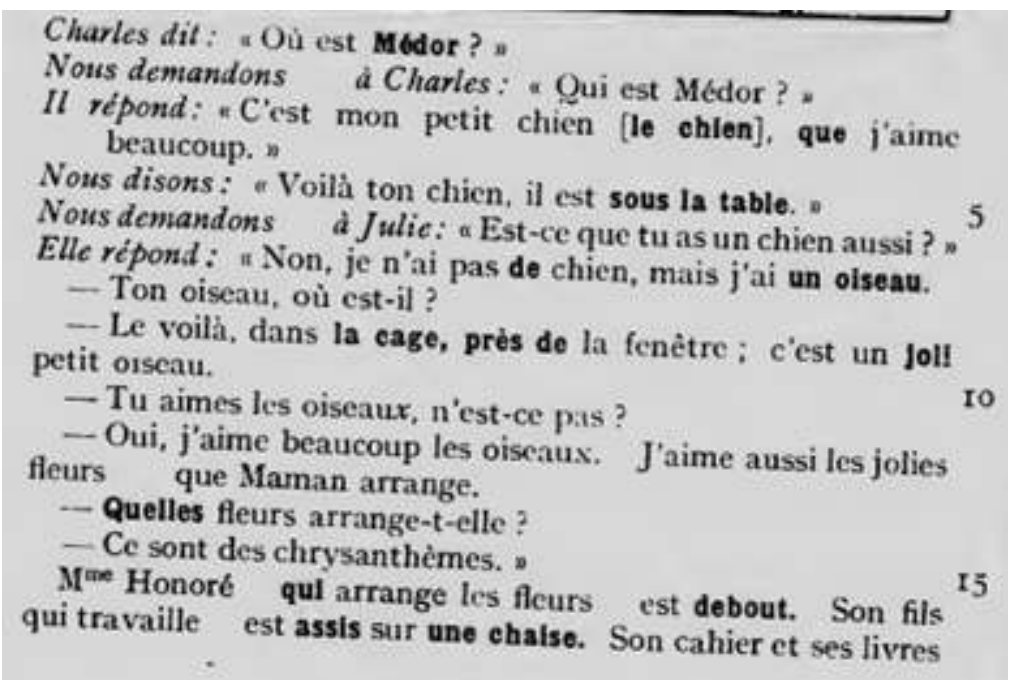

Document 9 : Rippmann (1915: 22)

Dans le manuel Further steps, Rippmann se sert des exercices de vocabulaire avec la consigne suivante: "Faites la description des images qui suivent». Donc, ici le principal objectif est de travailler encore une fois le vocabulaire en contexte et à partir de la gravure : 


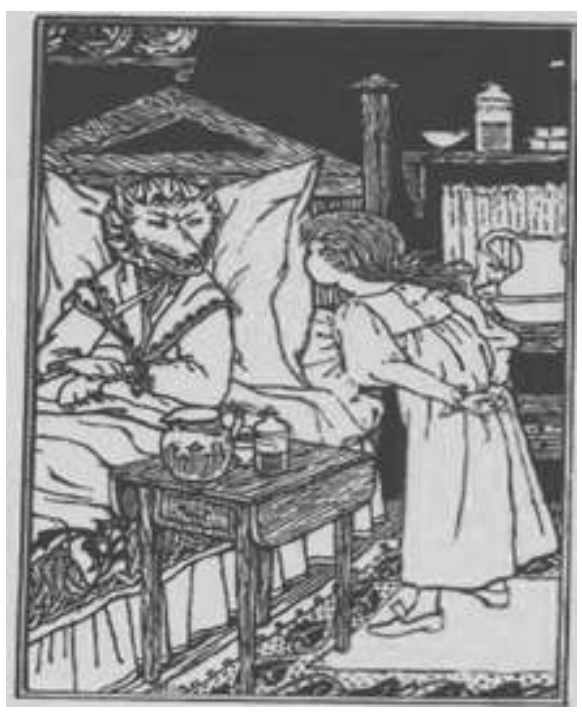

Document 10 : Rippmann (1916 : 138-139)

Pour ce qui est de leur manuel, Alge \& Rippmann (1904), utilisent également les images. Celles-là sont les mêmes qu'Alge avait utilisées dans son manuel. Les leçons s'articuleront pareillement à partir des gravures (voir document 2). Toutes les leçons et donc les exercices gravitent autour de ces tableaux. Au fur et à mesure que les leçons avancent, les textes qui ouvrent les leçons augmentent de longueur et alors les leçons deviennent plus complexes.

57 Valette se sert également des tableaux de Delmas pour illustrer le vocabulaire et construire la leçon et les activités en partant de ces images. Les activités proposées par cet auteur sont les activités de questions-réponses :

\section{Un Jour a'Hiver}

La neige, il neige. Ce tableau roprésente un jour d'hiver. Que ruprésente ce tableas? Que voyoz-vous sur les maisoni, sur les arbres, ot dans la rue? Jy vois de la neige. Do quello couleur est-elle? BTle ost blanobe. Est-olle chaude ou froide? Elle est froide.

La noige tombe du ciel. Drou tombe la neige? Quand tombe-t-elle? Elle tombe en hiver. Comment tombe-t-elle? Elle tombe on flocons. Quand la neige tombe, vous dites: il neige. Neige-t-il aujourdhui? Non, il ne neige pan. Quo font ces petits garçons? Ils font des boules de neige, ot ils ne battent.

f. La glace, il gèle. Voici uno rivikre. Voyéz-vous ees deux messieurs sur cotte rivièro? Oui, jo les vois ? ils marohent sur la rivière; ils putinent. Sur quoi patinent-ils ? Ils patinent sur ln riviere. Aveo quoi? Avea des patins. Ils peuvent patiner parce que ia rivitere est gelé (solide). Lo contraire de sotide est liguide. Leau est changbo on glace par le froid quand lo thermomitre est tris bas. En hiver il gele souvent. A Paris il ne gèle jamais en éte. Gele-t-il aujourd'hui ? Non, il ne gèle pas.

Le pont; traverser. Au-dessus de cetto rivilive vous voyez un pont. (Ma main droito ost our la table; ma main ganche est au-desere de la table.) Nous pansons sur lo pont, et nous traversons In rivière. Maintennat je tratered la classe. A Puris il est très diflicile de truverser les rues, parce qu'il $y$ a beancoup de voitures.

Document 11 : Valette $(1915: 67)$ 
De leur côté, Schweitzer \& Simonnot présentent également de petites images pour illustrer le vocabulaire, et des images qui représenteront des situations quotidiennes propres aux apprenants :

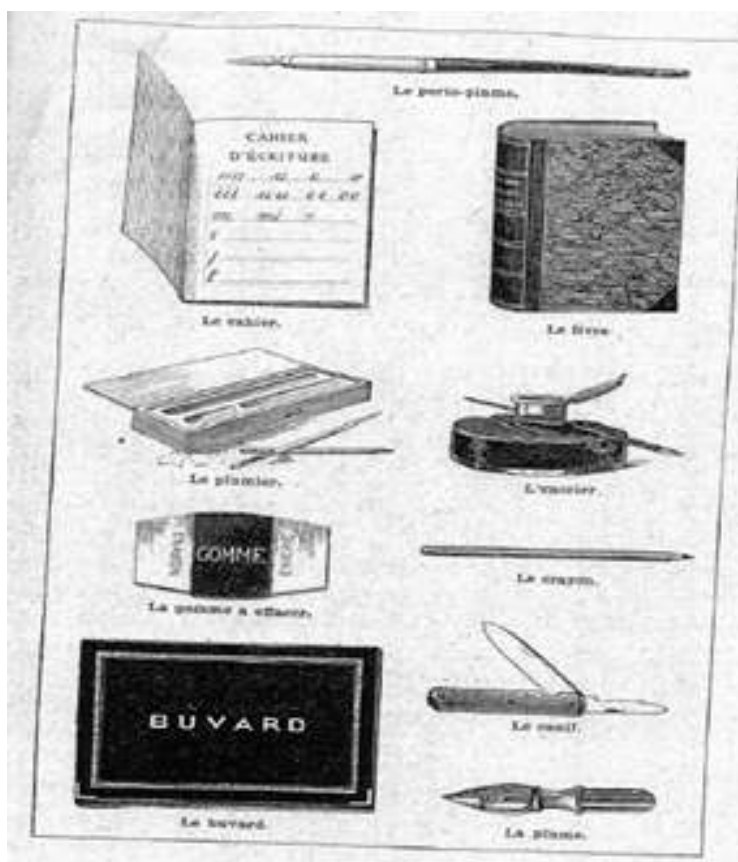

Document 12 : Objets appartenant à l'élève (Schweitzer \& Simonnot 1913 : 31)

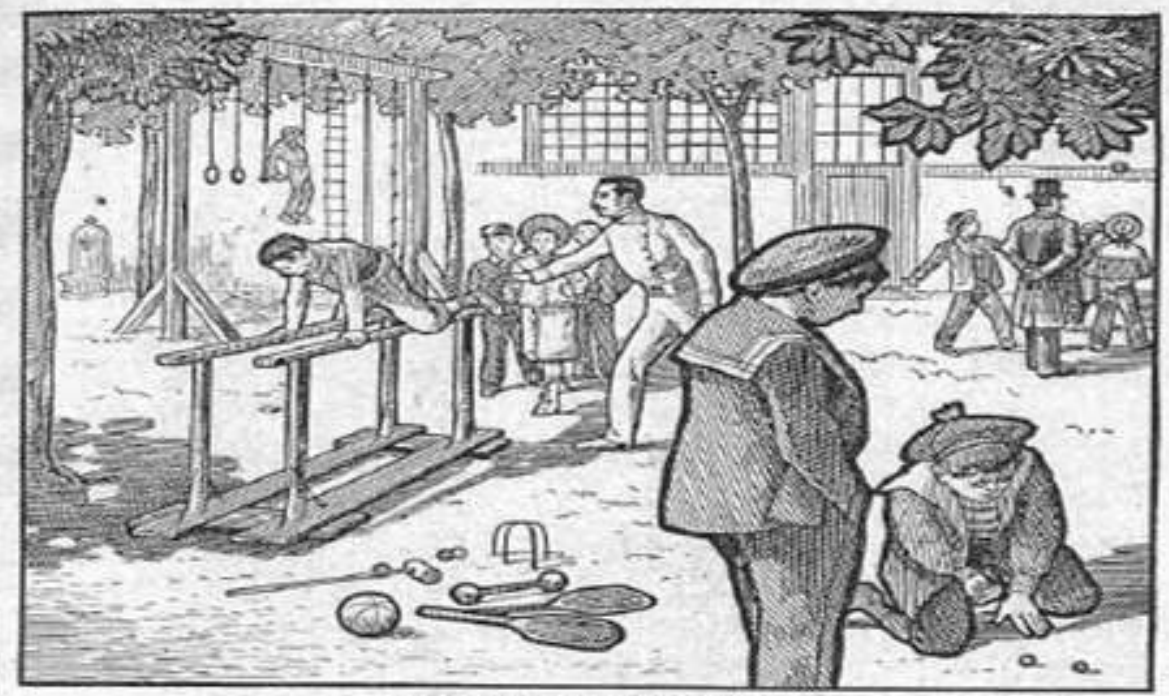

One cour te grmmantique.

Document 13 : Une cour de gymnastique (Schweitzer \& Simonnot $1913: 117$ )

Pour ces auteurs, le vocabulaire :

dans ce volume embrasse, avant tout, la vie scolaire. La classe est le milieu naturel où maîtres et élèves évoluent chaque jour et nouent entre eux des rapports qu'il importe tout d'abord de savoir exprimer dans la langue étrangère [...]. Dès que l'élève est capable de se mouvoir avec quelque aisance dans cette première sphère du langage, il passe aisément dans une sphère voisine. Il n'a plus qu'à couler de nouveaux vocables dans les moules des phrases typiques qui lui sont désormais familières (Schweitzer \& Simonnot 1913 : V-VI). 
Nous avons pu remarquer de quelle façon les images modélisent les manuels et sont la pierre angulaire de l'e/a du vocabulaire de la LE. La progression du vocabulaire est donc intimement liée à la progression des images. D'abord, il s'agit d'images simples (les objets de classe, les vêtements...) ; puis les images qui représentent les situations de la vie réelle des apprenants (les apprenants dans une cour de gymnastique, les apprenants avec leur famille...) sont plus complexes.

\section{La grammaire et le vocabulaire}

61 La MD présente la grammaire d'une façon implicite, sans la laisser de côté, car : « on ne saurait enfin prétendre que, dans notre méthode d'enseignement, la part de la grammaire soit sacrifiée. Pour s'en convaincre, il suffit de soumettre à un examen sérieux les parties du livre qui s'y rapportent [...], et l'on verra qu'il y a là de nombreux exercices écrits qui y ont trait » (Alge ${ }^{5} 1896 a$ : IX-X).

Les manuels analysés recommandent d'aller du connu à l'inconnu. La grammaire est principalement associée au vocabulaire, comme nous venons de le vérifier. Les auteurs de ces manuels insistent vivement de commencer l'e/a de la langue par le vocabulaire avec lequel la grammaire se marie.

Certains exercices de langage sont, en principe, des exercices de grammaire, puisque nous ne séparons pas la grammaire de l'étude du vocabulaire [...]. Mais, il y a tout un ordre d'exercices ayant plus spécialement en vue le maniement des formes grammaticales, tels les suivants: Mettre des substantifs au pluriel, au génitif singulier; changer les temps des verbes contenus dans un texte donné; former des phrases composées avec deux ou plusieurs propositions simples, etc. (Schweitzer 1903 : 30-31).

La présentation principale de la grammaire se fait à partir de petites phrases, de petits dialogues, où nous trouvons la grammaire en contexte, en nous éloignant définitivement de la grammaire théorique de la MT. Pour cette raison, les exercices principaux seront ceux qui représentent la grammaire plus le vocabulaire et ces exercices ne laissent rien au hasard: "les exercices s'enchaînent avec une rigueur absolue » (Rochelle $1906: 4$ ).

Le type d'exercices conseillé pour développer la grammaire sont: les questionsréponses, les activités de simulations de communication, ainsi que les exercices à compléter, les dictées, les rédactions de paragraphes :

La conversation qui était le but de notre premier enseignement devient un simple moyen d'augmenter le vocabulaire déjà acquis et de préciser les notions grammaticales au fur et à mesure que des formes nouvelles se présentent à nous. La lecture est, momentanément, notre nouveau but (Rochelle $1906: 24$ ).

Dans le manuel de la première année d'Alge (51896a: 92-128), les lectures suivies des questions, qui se trouvent à la fin de la partie grammaticale, aident à fixer les contenus grammaticaux préalablement travaillés. Les thèmes des lectures proposés par Alge, à titre d'exemple, sont les suivants : "La famille Bontemps ", " Notre maison ", " Notre village », «Les leçons de français », « Les écoles de St-Gall », « Notre maison d'école »... Rippmann, à son tour, introduit aussi une série de lectures, à la fin de son manuel, pour exercer la grammaire grâce à la lecture de ces petites histoires (Rippmann 1915 : 150-158).

66 Le manuel de la deuxième année d'Alge ( $\left.{ }^{5} 1896 \mathrm{~b}\right)$ est organisé ainsi, principalement, à partir de la morphologie verbale. Donc, pour travailler la grammaire, Alge propose une 
série d'exercices qui n'étaient pas présents dans la première année, tels que : complétez les phrases suivantes, citez, remplacez le présent par l'imparfait et complétez alors les phrases du thème précédent, rendez autrement les phrases suivantes... Il s'agit ici de l'application pratique des concepts grammaticaux théoriques.

Comme nous avons pu l'observer dans les manuels de Rippmann, les contenus grammaticaux à acquérir étaient notamment présents dans les exercices questionsréponses, et les termes grammaticaux soulignés en gras pour attirer l'attention des apprenants (cf. document 9). En outre, «Le cahier de Grammaire» complétait son manuel First steps où précisément les notions grammaticales étaient explicitement présentes en suivant la classification, d'un point de vue traditionnel. Cette partie reprend les notions grammaticales d'une série de leçons en guise de révision. Il est, également, question d'un "Cahier de Vocabulaire » organisé par groupes thématiques. Dans le deuxième manuel, les apprenants pourront y trouver des explications grammaticales relatives aux textes des leçons qui apparaissent en bas de page ainsi que des explications sur certains mots avec lesquels les apprenants pourraient avoir des difficultés :

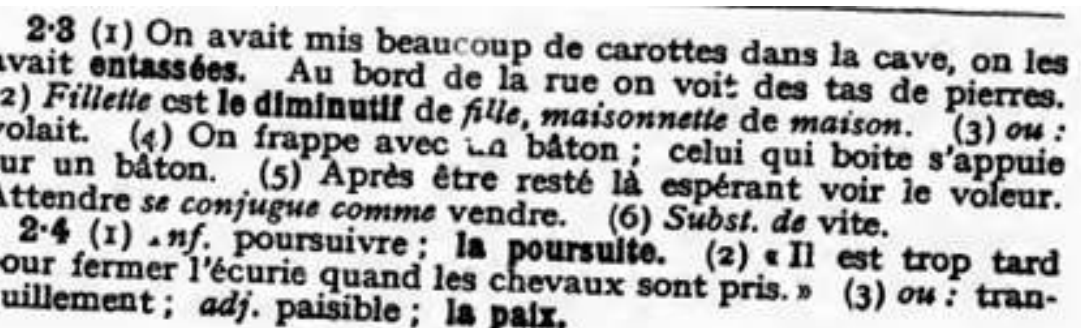

Document 14 : Rippmann (1916: 6)

Dans le manuel d'Alge \& Rippmann (1904), la présentation grammaticale suit l'ordre traditionnel et elle est organisée autour du vocabulaire présent dans les tableaux. Chaque petite leçon commence par un texte, où les mots grammaticaux seront en gras, et ensuite, il y aura des exercices sur ce texte, pour finir avec les explications grammaticales. Après avoir suivi quelques leçons, ces auteurs mettent à disposition de leurs apprenants un résumé grammatical, avec les explications de chaque notion grammaticale de toutes les leçons précédentes. Donc, une fois de plus, nous vérifions l'omniprésence de la grammaire dans la MD.

La grammaire est, alors, axée sur la progression du vocabulaire (sous forme de cercles concentriques autour de l'élève : la classe, la famille, le village, la campagne, la grande ville...) dans les manuels de Camerlynck \& Camerlynck. Pour ces auteurs, le verbe est "la clef de voûte de toute la construction grammaticale » (Camerlynck \& Camerlynck 1921 : V). Ainsi, selon eux, dans cette première année, il fallait «tracer les grandes lignes, en négligeant à dessein ce qu'on appelle les 'exceptions'; et nous nous sommes attaqués avant tout aux difficultés fondamentales du français pour les débutants » (ibid. : V).

Dans le manuel de la deuxième année, ces auteurs ( $\left.{ }^{5} 1930\right)$ ajoutent un «memento grammatical» qui permettra aux apprenants de retrouver, sous une forme méthodique, les notions acquises intuitivement dans le cours de l'ouvrage. Ils sont pleinement conscients que la MD ne peut pas négliger la grammaire : « Nous n'avons eu garde de négliger une fée, moins souriante et moins enguirlandée que les autres, mais qui se venge toujours cruellement quand on oublie de la convier à la fête; nous voulons 
parler de Dame Grammaire » (Camerlynck \& Camerlynck 1930 : 6). À titre d'illustration, les exercices trouvés dans ces manuels pour travailler la grammaire sont des exercices de conjugaison et des parties grammaticales :

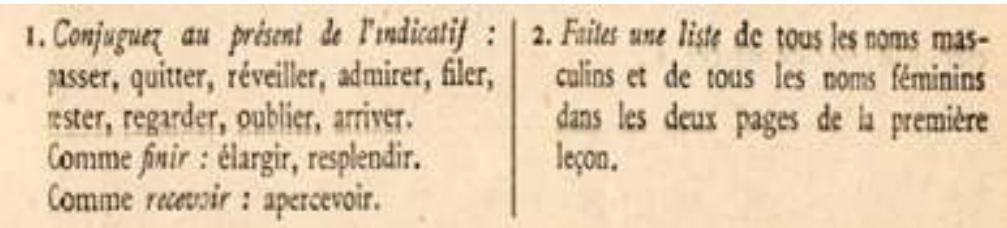

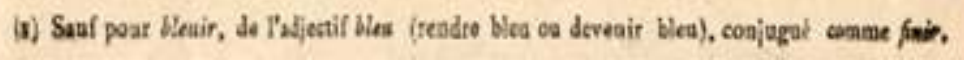

Document 15 : Camerlynck \& Camerlynck (1930 : 9)

71 Quant aux manuels de Valette, la grammaire est la clé de cette expérience directe pour apprendre la LE et les explications grammaticales font également partie du discours de ces manuels :

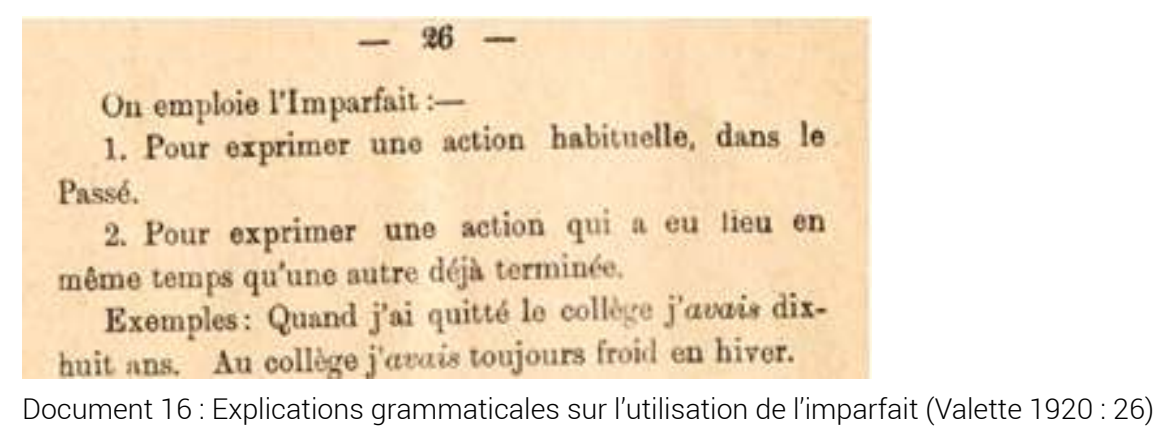

En outre, les exercices d'application grammaticale suivent normalement le modèle de question-réponse qui reprendra le vocabulaire utilisé dans la leçon ainsi que les notions grammaticales :

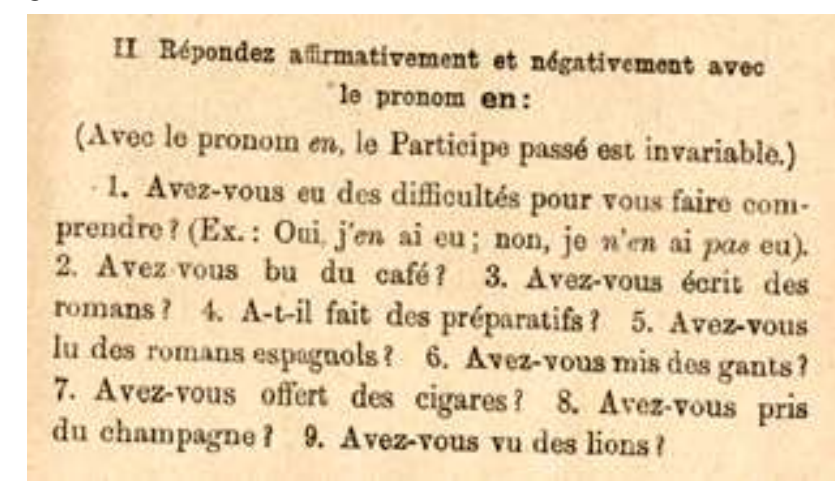

Document 17 : Valette $(1920: 11)$

73 Pour Schweitzer \& Simonnot (1913), la grammaire est « l'âme même de la matière que nous traitons et forme avec celle-ci un tout organique. C'est la grammaire apprise sur le vif, sans théorie préalable. Les règles découlent naturellement de nos phrases-types logiquement graduées » (Schweitzer \& Simonnot 1913 : VI). Nous nous rendons compte, encore une fois, que la grammaire est toujours présente dans ces manuels. Et donc, « la grammaire est ainsi remise à sa place : c'est la constatation de l'usage » (Schweitzer \& Simonnot 1913 : VI, en italique dans le texte).

74 Par exemple, ces auteurs expliquent, de façon détaillée la manière de faire acquérir la morphologie qui est liée au vocabulaire : 
Tous les faits essentiels de morphologie sont intimement liés à l'étude du vocabulaire et s'enseignent au moyen de la méthode directe plus facilement et d'une manière plus vivante que par les explications abstraites d'un livre. Les faits de syntaxe proprement dits ou de construction peuvent être interprétés avec tout autant de facilité par voie intuitive. Il suffit, en effet, d'écrire chaque phrase type au tableau pour faire voir la place des différents mots dans les divers genres de propositions. C'est une simple question d'ordre qui fait passer l'élève progressivement des formes les plus simples aux plus complexes (Schweitzer \& Simonnot $1917:$ 153).

Ils sont conscients que les apprenants ne sont pas une tabula rasa par rapport à l'e/a de la grammaire :

Il ne faut pas perdre de vue que nos élèves nous arrivent déjà avec une certaine culture grammaticale dans leur langue maternelle et que, par suite, la question se ramène à mettre de nouvelles étiquettes sur des idées abstraites qu'ils possèdent déjà (Schweitzer \& Simonnot $1917: 164$ ).

En résumé, laisser de côté complètement la grammaire dans la MD n'aurait aucun sens, comme nous venons de le constater dans ces manuels, puisqu'il s'agit d'un élément constitutif de l'étude de la LE. Or, comme nous avons pu le constater, «le choix des points grammaticaux (à pratiquer) paraît être basé sur l'intuition de l'enseignant ou des concepteurs du matériel didactique » (Germain 1993 : 129).

\section{Les résultats}

Après avoir analysé ces manuels, nous constatons, d'abord, la présence de la MD en tant que courant méthodologique de son époque, et ensuite, la symbiose entre la grammaire et le vocabulaire dans l'e/a de la LE.

Ces différents manuels ont en commun l'application de la MD pour l'e/a de la langue française par un public scolaire. La plupart des manuels présentent les caractéristiques suivantes : le rejet de la LM, la présence des images/gravures, les exercices destinés à (ré)employer la langue à apprendre, la construction de la progression grammaticale autour du vocabulaire.

79 Tous les auteurs centrent la progression grammaticale sur le vocabulaire et sur les images/gravures. Celles-ci représentent, en partie, le vocabulaire et la grammaire à acquérir. Au fur et à mesure que les leçons deviennent plus complexes et que les apprenants ont déjà acquis les notions les plus simples, les images commencent à disparaître et les manuels préconisent une progression plus 'théorique', en suivant pour ainsi dire la MT. L'idée partagée selon laquelle dans la MD la grammaire est déconseillée ne se retrouve pas dans les manuels analysés, où la grammaire est bien présente, mais axée sur la progression du vocabulaire de l'e/a de la LE (Alge, Rippmann, Camerlynck \& Camerlynck, Alge \& Rippmann, Valette, Schweitzer \& Simonnot).

\section{En guise de conclusion...}

L'analyse de ces manuels nous a fait, définitivement, abandonner l'idée, encore présente, selon laquelle, la grammaire ne faisait pas vraiment partie de l'e/a des langues étrangères dans la MD. À notre avis, la progression grammaticale qui y est 
préconisée - essentiellement sous une perspective pratique -, pourrait être décrite comme l'état embryonnaire de la grammaire en contexte.

81 Si l'on prend en compte toutes ces considérations, nous pouvons résumer la principale caractéristique de la MD par ce proverbe : "c'est en forgeant qu'on devient forgeron ». La spécificité fondamentale de cette MD est, en conséquence, la pratique pour pouvoir communiquer dans une LE.

BIBLIOGRAPHIE

\section{Sources primaires}

ALGE, Sines ( $\left.{ }^{5} 1896 a\right)$. Leçons de français, première année. St. Gall : Librairie Fehr.

ALGE, Sines ( $\left.{ }^{5} 1896 \mathrm{~b}\right)$. Leçons de français, deuxième année. St. Gall : Librairie Fehr.

ALGE, Sines \& RipPMANN, Walter. $\left({ }^{9} 1904\right)$. Leçons de français basées sur les tableaux de Hölzel. St. Gall :

Librairie Fehr.

CAMERLYNCK, Gabrielle-Jeanne \& CAMERLYNCK, Gustave-Henri (1921). Première année de français.

France. Méthode directe de français avec notation phonétique. Boston, New York... : Allyn and Bacon.

CAMERLYNCK, Gabrielle-Jeanne \& CAMERLYNCK, Gustave-Henri ( $\left.{ }^{5} 1930\right)$. France $: 2^{e}$ année de français.

Paris : Didier.

RIPPMANN, Walter (1915). First steps in French. London, Toronto : J. M. Dent, Sons Limited.

RIPPMANN, Walter (1916). Further steps in French. London, Paris, Toronto : J.M. Dent, Sons Limited.

SCHWEITZER, Charles \& SIMONNOT, Émile (1913). Méthode directe pour l'enseignement de la langue française. Première année. Paris : Librairie Armand Colin.

VALETTE, Marc de (1914). La méthode directe pour l'enseignement des langues vivantes à l'aide des tableaux auxiliaires. $1^{\text {er }}$ livre. Paris : Hachette.

VALETTE, Marc de (1920). La méthode directe pour l'enseignement des langues vivantes à l'aide des tableaux auxiliaires. $2^{e}$ livre. Paris : Hachette.

\section{Sources secondaires}

BÉNÉDICT, Gaston (1950). L'enseignement vivant des langues vivantes par la méthode directe progressive. Lausanne : Éditions Pro Schola.

BESSE, Henri (2012). « Éléments pour une archéologie de la méthode directe ». Documents pour l'histoire du français langue étrangère ou seconde, 49, 11-30.

FERNÁNDEZ FRAILE, Mª Eugenia \& SUSO LóPEZ, Javier (1999). La Enseñanza/Aprendizaje del francés en España entre 1767 y 1936: objetivos, contenidos y procedimientos. Granada : Universidad de Granada. 
GERMAIN, Claude (1993). Evolution de l'enseignement des langues : 5000 ans d'histoire. Paris : CLE International.

JESPERSEN, Otto (1904). How to teach a foreign language. London : Swan Sonnenschein \& Co. Ltd ; New York : The MacMillan Co.

JESPERSEN, Otto (1925). « La enseñanza de las lenguas extranjeras ». Boletín de la Institución libre de enseñanza, 788-789, 321-370.

JESPERSEN, Otto (1926). « La enseñanza de las lenguas extranjeras (continuación) ». Boletín de la Institución libre de enseñanza, 790, 5-9.

REINFRIED, Marcus (1990). « Les origines de la méthode directe en Allemagne ». Documents pour l'histoire du français langue étrangère ou seconde, 6, 126-157.

REINFRIED, Marcus (1999). « Le mouvement réformiste et la méthode directe en Allemagne : développement, fondement théorique, variations méthodologiques ». Documents pour l'histoire du français langue étrangère ou seconde, 23. En ligne : [http://journals.openedition.org/dhfles/3045] [consulté le 15.11.19]

ROCHELLE, Ernest (1906). La méthode directe dans l'enseignement des langues vivantes. Bordeaux : Delmas.

SCHWEITZER, Charles (1903). Méthodologie des Langues Vivantes. Notes prises aux Conférences faites à la Sorbonne. Paris : Armand Colin.

SCHWEITZER, Charles \& SIMONNOT, Émile (1917). Méthodologie des langues vivantes. Paris : Armand Colin.

SUSO LóPEZ, Javier \& FERNÁNDEZ FRAILE, Mª Eugenia (2008). Repertorio de manuales para la enseñanza del francés en España (siglo XX). Granada : Comares.

SuSO LóPEZ, Javier \& VALDÉS MELGUIZO, Irene (2020). « La grammaire dans le mouvement de la réforme en France et en Grande-Bretagne ». In Simon Coffey (dir), The History of Grammar in Foreign Language Teaching. Amsterdam : Amsterdam University Press, 191-210.

SUSO LÓPEZ, Javier (2016). Diccionario de historia de la enseñanza del francés en España (siglos XVI-XX), entrée Méthode Directe. Groupe de recherche en Linguistique Appliquée. Éditeur principal : Juan Francisco GARCIA BASCUÑANA. Tarragona : Université Rovira i Virgili. En ligne : [http:// www.grelinap.recerca.urv.cat/ca/projectes/ diccionariohistoria-ensenanza-frances-espana/].

SWEET, Henry ([1899]1900). The practical study of language: a guide for teachers and learners. New York : Henry Holt. En ligne : [https://archive.org/details/practicalstudyl00sweegoog/page/n150] [consulté le 20.09.19].

VIËTOR, Wilhelm (1882). Der Sprachunterricht muss umkehren! Ein Beitrag zur Überbürdungsfrage von Quousque Tandem. Heilbronn : Gebr. Henninger.

\section{RÉSUMÉS}

Dans cette étude, nous analysons une série de manuels publiés entre 1896 et 1930 (Alge ; Alge \& Rippmann ; Camerlynck \& Camerlynck ; Rippmann ; Schweitzer \& Simonnot ; Valette) qui représentent la méthode directe en nous centrant sur la place de la grammaire. Nous constatons qu'il existe une certaine cohérence parmi tous les manuels analysés dans leur rapport à la 
représentation grammaticale. La progression grammaticale se fait principalement à travers le vocabulaire et les images/gravures, donc, par la pratique. Cette progression grammaticale de la méthode directe se dissocie définitivement de la progression préconisée par la méthode traditionnelle, où la grammaire est le fil rouge dans l'enseignement et l'apprentissage des langues étrangères.

This article examines a number of textbooks on the direct method published between 1896 and 1930 by Alge, Alge \& Rippmann, Camerlynck \& Camerlynck, Rippmann, Schweitzer \& Simonnot, and Valette. In particular, the present research focuses on the grammatical approach adopted by these works. This analysis identifies a certain consistency among all the textbooks examined in relation to grammatical representation. This is so because grammatical progression is mainly performed through vocabulary and images/engravings - hence, through practice. According to the direct method, grammatical progression definitively dissociates from the progression recommended by the traditional method, where grammar remains the common thread in the teaching and learning of foreign languages.

INDEX

Mots-clés : méthode directe, progression grammaticale, manuels XIXe-XXe siècles

Keywords : direct method, grammatical progression, textbooks 19th- 20th centuries

\section{AUTEUR}

IRENE VALDÉS MELGUIZO

Université de Grenade - ivaldes@ugr.es 